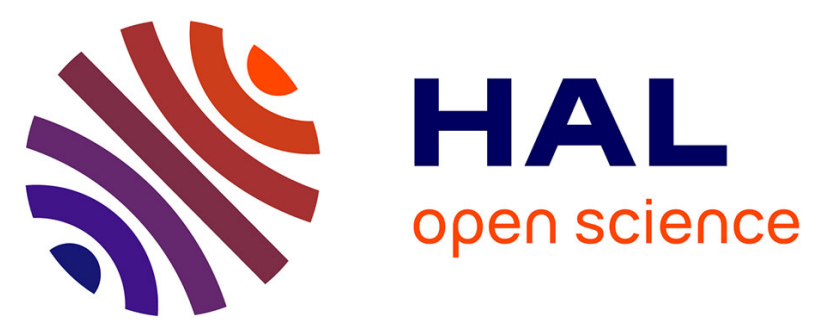

\title{
Creative competencies and cognitive processes associated with creativity are linked with positive schizotypy
}

Jennifer Jacquet, Lionel Delpech, Jonathan Bronchain, Patrick Raynal

\section{To cite this version:}

Jennifer Jacquet, Lionel Delpech, Jonathan Bronchain, Patrick Raynal. Creative competencies and cognitive processes associated with creativity are linked with positive schizotypy. Creativity Research Journal, 2020, 32 (2), pp.142-150. 10.1080/10400419.2020.1733895 . hal-03191087

\section{HAL Id: hal-03191087 \\ https://hal.science/hal-03191087}

Submitted on 6 Apr 2021

HAL is a multi-disciplinary open access archive for the deposit and dissemination of scientific research documents, whether they are published or not. The documents may come from teaching and research institutions in France or abroad, or from public or private research centers.
L'archive ouverte pluridisciplinaire HAL, est destinée au dépôt et à la diffusion de documents scientifiques de niveau recherche, publiés ou non, émanant des établissements d'enseignement et de recherche français ou étrangers, des laboratoires publics ou privés. 
Creative competencies and cognitive processes associated with creativity are linked with positive schizotypy

Jennifer Jacquet, Lionel Delpech, Jonathan Bronchain, Patrick Raynal

Centre d'Etudes et de Recherches en Psychopathologie et Psychologie de la Santé, Université de Toulouse, UT2J, France

Author notes

The authors attest that the data reported here are not used in any other publications and that there are no conflicts of interest. This research did not receive any specific grant from funding agencies in the public, commercial, or not-for-profit sectors.

Corresponding author: Patrick Raynal, Laboratoire CERPPS, Université de Toulouse-Jean Jaurès, 5 allées Antonio Machado, 31058 Toulouse, France. Phone number: +33 5615035 59. Fax number: +33 5612570 93. E-mail: patrick.raynal@inserm.fr 


\begin{abstract}
The links between schizotypy and creativity remain ill-defined. This study assessed creative competencies and cognitive processes associated with creativity in groups of individuals with distinct levels of schizotypy dimensions. Groups were obtained through cluster analysis of a large student sample $(N=946)$. All dimensions of creative competencies (Capturing, Broadening, Surrounding, Challenging) were higher in a pure positive schizotypy cluster than in clusters characterized by negative schizotypy or low schizotypy. Regarding creative processes associated with creativity, two dimensions, namely Imagery and Incubation, were higher in the pure positive schizotypy cluster than in negative schizotypy or low schizotypy clusters, while other dimensions (Brainstorming, Idea manipulation, Imagery, Incubation) were higher in positive schizotypy cluster when compared with negative schizotypy groups. This supports the notion that creative cognitive processes are more developed in positive than in negative schizotypy. Most interestingly, it also suggests that positive schizotypy can be beneficial even when compared with non-schizotypal individuals, since all dimensions of creative competencies and two dimensions of creative cognitive processes were higher in individuals with high positive schizotypy than in those with low schizotypy. This could have implications to improve the difficult and stigmatizing personal experience of individuals with high positive schizotypy about their own symptoms.

Keywords: Creative competencies; Cognitive processes associated with creativity; Positive schizotypy; Cluster analysis.
\end{abstract}


Creative competencies and cognitive processes associated with creativity are linked with positive schizotypy

Although elevated schizotypy may predispose individuals to a psychotic disorder (van Os \& Linscott, 2012), a debate is currently ongoing about the possible advantage(s) of this personality trait. Schizotypy is commonly assessed using questionnaires assessing symptoms related with those of schizophrenia, and individuals scoring high in such questionnaires were found to have an enhanced risk for psychosis (van Os \& Linscott, 2012; Kwapil \& BarrantesVidal, 2015). Schizotypy is considered as a multidimensional construct encompassing positive, negative, and disorganized traits. Positive schizotypy describes perceptual aberrations similar to subsyndromal hallucinations, as well as unusual ideas which may be considered as attenuated forms of overinvested ideas related with delusions of schizophrenia. Negative schizotypy involves a reduction in emotional, physical and social functions, such as the experience of pleasure of interest in social contacts. The disorganized dimension includes thought disorder and bizarre behavior (Ettinger et al., 2015; Fonseca-Pedrero et al., 2017).

On the other hand, one may wonder whether schizotypy might have some advantages, allowing the persistence of this trait over generations (Keller \& Miller, 2006). This question led to studies of health and wellbeing in individuals with high schizotypy. As a whole, they showed that all dimensions of schizotypy are linked to lower life satisfaction and higher negative affect, when compared with individuals scoring low in schizotypy (Cohen \& Davis, 2009; Abbott, Do, \& Byrne, 2012). However, these associations are weaker in individuals displaying high scores of positive schizotypy and low levels of negative and disorganized dimensions, which led to the concept of "healthy schizotypy" (Mohr \& Claridge, 2015). Indeed, these individuals showed favorable subjective and psychological wellbeing, comparable to subjects with low schizotypy (Goulding, 2004; Tabak \& de Mamani, 2013). Other studies linked positive schizotypy to pleasant and enriching mental experiences (McCreery \& Claridge, 2002; Schofield \& Claridge, 
2007) or to a higher level of perceived social relationships (Raynal, Goutaudier, Nidetch, \& Chabrol, 2016).

In addition, a link between positive schizotypy and creativity has been suggested (Richards, 2001; Sass \& Schuldberg, 2001). Creativity can be defined as the ability to produce something new and worthwhile, a new solution to a problem, a new method or device, or a new artistic object (Mumford, 2003). The hypothetical association of positive schizotypy and creativity is based initially on studies reporting correlations between artistic creativity and features of personality assessed as positive schizotypy. Indeed, visual artists and poets were characterized by significant features of positive schizotypy and a low level of introvertive anhedonia, a component of negative schizotypy (Nettle, 2006). In addition, using a sample of 100 artists from different domains, traits of positive schizotypy were the strongest predictor of creative experiences, when compared with Big Five personality traits (Nelson \& Rawlings, 2010). However, in a student sample, intelligence and openness (a Big Five personality trait) were better predictors of creativity than schizotypy (Miller \& Tal, 2007). Creativity tests were also used to address the link between schizotypy and creativity. In a verbal fluency test, college students with high positive schizotypy had better performances than those with elevated negative schizotypy (Tsakanikos \& Claridge, 2005). Similarly, using a self-rating creativity test and measurements of creative achievements, creativity scores were higher in individuals with high positive schizotypy, when compared with those with high negative schizotypy (Batey \& Furnham, 2008). In addition, studies provided evidence of a common neural basis between creativity and schizotypy (Park, Kirk, \& Waldie, 2015).

However, other studies reported divergent results. For example, a differentiation was established on a neuropsychological basis between personality profiles characterized as "creative" or "schizotypal", each profile involving distinct executive functions processing semantic information (Fisher, Heller, \& Miller, 2013). In another study, high schizotypal individuals displayed increased performance in creativity tasks, which was mediated by higher 
overinclusive thinking, i.e. greater inability to preserve conceptual boundaries (Wang et al., 2018). However, in this study, better creative performance was also mediated by higher cognitive inhibition, which is somewhat dissonant with the literature on the link between cognitive inhibition and creativity (Radel, Davranche, Fournier, \& Dietrich, 2015). Therefore, further studies are required to explore the links between creativity and schizotypy.

The field of creativity research has been generally poor in direct measures of creativity but recently, self-report measures of creative processes have been developed. Of note, Epstein, Schmidt, and Warfel (2008) proposed a reliable scale, the Epstein Creativity Competencies Inventory for individuals (ECCIi), based on the four following core competencies of creative expression: 1-Capturing: preserving new ideas as they occur, finding places and times where new ideas can be observed easily, using dreams and daydreams as sources of ideas; 2-Challenging: taking on difficult tasks, setting open-ended goals, managing fear and stress associated with failure effectively; 3-Broadening: seeking training, experience, and knowledge outside current areas of expertise; 4-Surrounding: changing physical and social environments regularly, seeking out unusual stimuli.

In addition, the Cognitive Processes Associated with Creativity (CPAC) scale was developed to address the cognitive strategies used during creative processing. These strategies include brainstorming, perspective-taking, metaphorical and analogical thinking, incubation, imagery/sensory, and flow (Miller, 2014). Idea generation (brainstorming) refers to the attempt to generate as many potential responses or solutions as possible, regardless of the plausibility. Idea manipulation (perspective-taking) can be described as an intentional attempt at perceptual transformation with a shift in perspective allowing the individual to conceptualize or understand the situation in a different way. Metaphorical and analogical thinking is about taking ideas from one context and applying them in a new setting, producing a connection between the current problem and a similar or related situation. Incubation refers to a mental process taking place while the person is engaged in other activities, allowing unconscious testing of idea associations 
which may be relevant to the problem. Imagery/sensory refers essentially to internal visualization as a key element of the creative process, and to any type of sensory modality (auditory, tactile etc.) that can be connected to creativity. Flow can be defined as an almost automatic yet highly focused state of consciousness that occurs when an individual is engaged in intense work, often of a creative nature (Csikszentmihalyi, 2014).

The aim of this study was to take advantage of novel reliable creativity scales, namely ECCIi and CPAC, to further explore whether creativity is a beneficial outcome of schizotypy and to define the underlying processes. A student sample was studied using self-report questionnaires, which allowed to classify individuals based on their scores of distinct schizotypal dimensions. Our first hypothesis was the possibility of identifying different clusters of participants based on schizotypy dimensions. A subsequent hypothesis was that these groups could be distinguished from each other using different dimensions of competencies of creative expression and of cognitive processes associated with creativity.

\section{Method}

\section{Participants}

The data were collected through an online survey that was distributed to students from different universities in France. The link was shared on social networks in groups specifically dedicated to students. The sample was composed of 946 students aged between 18 and 26 who completed the questionnaire ( $83 \%$ female, $17 \%$ male). The mean age of the sample was 21.17 years $(S D=1.95)$. The objectives of the study were presented at the beginning of the online questionnaire and the survey was presented to participants as a study of creativity and personality in students. Each of the participants had to give her/his informed consent. Participants were informed that their answers to the questionnaires would remain anonymous and confidential. The study followed the guidelines of the Helsinki declaration. No compensation was offered to participate in the study. 


\section{Measures}

\section{Creativity Competencies}

Creative competencies were assessed using the Epstein Creativity Competencies Inventory for Individuals (ECCIi; Epstein et al., 2008). This scale contains 28 items assessing 4 measurable competencies: Capturing (item sample: "I always keep a recording device by my bed at night"), Challenging (e.g., "When I set goals for myself, I make sure they're open-ended"), Broadening (e.g.,"I often read books from outside my specialty"), and Surrounding (e.g., "I redecorate or rearrange my work environment regularly"). Answers were recorded on a Likert scale (from $1=$ totally disagree to 5 = totally disagree). In Epstein \& Phan (2012), Cronbach's alpha was .85 and the mean total score of a sample from the general population from US and Canada was 84.42 (SD is not available).

\section{Creative cognitive processes}

These were measured using the Cognitive Processes Associated with Creativity (CPAC) scale (Miller, 2014). This instrument assess the six following dimensions of cognitive processes involved in creativity: Idea generation (item sample: "While working on something, I try to generate as many ideas as possible"), Metaphorical/analogical thinking (e.g., "If I get stuck on a problem, I make connections between my current problem and a related situation"), Idea manipulation (e.g., "Combining multiple ideas can lead to effective solutions"), Incubation (e.g., "When I get stuck on a problem, a solution just comes to me when I set it aside"), Imagery/sensory (e.g., "If I get stuck on a problem, I visualize what the solution might look like") and Flow (e.g.,."I can completely lose track of time if I am intensely working"). The CPAC scale contains 28 items rated with a Likert scale (from $1=$ never to $5=$ always). In Miller's study (2014) Cronbach's alpha was .86 and the mean total score of undergraduate students in Psychology was $99.59(\mathrm{SD}=9.87)$.

\section{Schizotypy}


Schizotypal traits were assessed using a French version of the Schizotypal Personality Questionnaire-Brief (SPQ-B), a 22-item self-administered scale (Raine \& Benishay, 1995; Bronchain, Chabrol, \& Raynal, 2018). Items were rated on a 4-point Likert type scale ranging from 1 (strongly disagree) to 4 (strongly agree). An item example is "People sometimes find me aloof and distant". The three following dimensions of schizotypy are assessed using three subscales: cognitive-perceptual deficits ("Positive"), interpersonal deficits ("Negative") and disorganization. A high total score is indicative of elevated schizotypal traits. Cronbach's alpha was .89 in a former study (Bronchain, Chabrol, \& Raynal, 2018).

\section{Anxiety and depression symptoms}

These were measured using the Hospital Anxiety and Depression Scale (Zigmond \& Snaith, 1983). This questionnaire contains 14 items, half of them dedicated to measuring depressive symptoms (e.g., "I feel as if I am slowed down"), and the other half to anxiety (e.g., "I feel tense or wound up"). Items were rated on a 4-point Likert scale.

\section{Satisfaction with life}

Satisfaction with life was assessed using the Satisfaction with Life Scale, a 5-item scale (e.g., "In most ways my life is close to my ideal") scored from $1=$ strongly disagree to $7=$ strongly agree (Diener, Emmons, Larsen, \& Griffin, 1985).

\section{Subjective well-being}

Subjective well-being was measured using three subscales (positive relations, selfacceptance and personal growth; each contains 7 items) of the Psychological Well-Being Scale (Ryff, 1989). An item example is: "Most people see me as loving and affectionate". Responses are scored on a scale ranging from 1 (strongly disagree) to 6 (strongly agree). 


\section{Results}

\section{Descriptive statistics}

Descriptive statistics are shown in Table 1 (left part). Cronbach's alpha values for schizotypy and creativity scales (CPAC and ECCIi) were above .80 , reflecting good internal consistency of these instruments.

\section{Factor analysis to identify schizotypy dimensions}

A factor analysis of SPQ-B was performed based on the study by Compton et al. who suggested caution with respect to the proposed SPQ-B subscales and recommended to use factor analytic techniques to study the SPQ-B latent structure in specific samples (Compton, Goulding, Bakeman, \& McClure-Tone, 2009). To identify the different factors of schizotypy in this sample, a principal component analysis was conducted on the 22 SPQ-B items. The eigenvalue curve suggested either a 3- or 4-factor solution. The 4-factor solution was retained as it accounted for $52 \%$ of the total variance. Each item was assigned to one of the factors if this item loaded greater than .30 on that factor and if there was a difference of at least .20 between the loading of this item for this factor and for any other factors (Table 2). The factors were called "Negative schizotypy" (5 items, e.g., "unable to get close to people"), "Social anxiety" (4 items, e.g., "very uneasy talking to people"), "Positive schizotypy" (3 items, e.g., "sense some person or force") and "Eccentricity" (3 items, e.g., "unusual mannerism and habits").

\section{Cluster analysis}

Based on the four factors of schizotypy identified above, cluster analysis was conducted in two steps to generate profiles. Absence of multicollinearity was evaluated through the correlations between the variables selected for this analysis (all variables had tolerance values $>$ .65). In the first step, a hierarchical cluster analysis was performed (Ward's method with squared Euclidean distance). The agglomeration schedule and dendrogram were used to identify the number of clusters. The dendrogram is presented in Figure 1. The agglomeration schedule 
showed a sudden increase in linkage distance (from 74 to 182) when four clusters merged to three clusters. This confirmed that the passage from four to three clusters would have more impact on the heterogeneity of the clusters than previous stages of the analysis. Therefore, the four cluster solution was the most appropriate. In the second step, K-means clustering was used to assign individuals to one of the identified clusters. A discriminant analysis showed clear differences between clusters (Wilks' $\lambda=.108, p<.001$ ) with $97.3 \%$ of cases correctly classified.

Figure 2 represents the mean (z-score) of each schizotypy factor for the four following clusters: a group called "High schizotypy" (H, $n=174,18 \%)$ aggregates participants with scores higher than the mean by about one standard deviation (SD) value when considering any schizotypy dimensions; A "Low schizotypy" cluster (L, $n=237,25 \%)$ gathers participants with scores of the four schizotypy factors lower than the mean by nearly one SD value; Another cluster called "Positive and Eccentric" (PE, $n=255,27 \%)$ is composed of participants with scores higher than the mean by about half SD regarding the Positive and Eccentricity dimensions, while scoring below the mean for the Negative and Social anxiety factors; The fourth cluster was called "Negative and Social anxiety" (NS, $n=280,30 \%$ ), as it includes individuals with scores higher than the mean by about half SD regarding the Negative and Social anxiety dimensions, while scoring below the mean for the Positive and Eccentricity dimensions.

These clusters were then compared to each other using ANOVA (Table 1, right part). Significant differences were found between clusters regarding all schizotypy factors, and effect sizes (eta squared) were strong, which further validated the classification. It was also verified that psychopathology levels were consistent with cluster features (i.e., high levels of negative schizotypy were associated with higher level psychopathology and lower level of well-being). Indeed, anxiety or depressive symptoms were more elevated in H and NS than in L or PE, while, consistently, life satisfaction and well being were lower in H and NS than in L and PE (effect sizes in the small-medium range). 
Following these controls, cluster comparison was achieved for creativity scales. H and PE displayed significantly higher scores of creativity competencies (ECCIi) when compared with NS and L, suggesting that creativity was associated with clusters scoring high in positive schizotypy. In terms of creative cognitive processes (CPAC), both $\mathrm{H}$ and PE scored significantly higher than NS, while PE scored higher than L. These results thus suggested that individuals with elevated positive schizotypy (H or PE) display a higher level of cognitive processes associated with creativity, when compared with individuals characterized by negative schizotypy (NS).

Next, clusters were compared based on scores of creative competencies subscales (ECCIi subscales). H and PE scored significantly higher than L and NS when considering Capturing, Broadening and Surrounding (Table 3). Regarding Challenging, the $\mathrm{H}$ group scored similarly to L and NS, and significantly lower than PE, suggesting that enhanced Challenging is only associated with "pure" positive schizotypy (i.e., individuals with elevated positive schizotypy and low negative schizotypy, such as in PE).

Finally, clusters were compared with respect to subscales of creative cognitive processes (CPAC subscales). Of note, the pure positive schizotypy cluster PE scored significantly higher than the negative schizotypy cluster NS in 4 out of 6 subscales (Idea manipulation, Imagery, Idea generation and Incubation). In addition, PE subscores for Imagery or Incubation were significantly more elevated than those of low schizotypes (L). Effect sizes were small for variables of creativity measures.

\section{Discussion}

This study assessed creative competencies and cognitive processes associated with creativity in a large student sample distributed in four groups, each displaying distinct levels of schizotypy dimensions, namely a high schizotypy cluster $(\mathrm{H})$, a "pure" positive schizotypy cluster (PE), a "pure" negative schizotypy cluster (NS), and a low schizotypy group (L). Comprehensive characterization of these groups, in terms of schizotypy factors, psychopathology and well-being, 
was achieved using factor analysis and cluster analysis, which then allowed to compare groups regarding competencies and cognitive processes associated with creativity.

When considering creative competencies measured by ECCIi, the two clusters high in positive schizotypy ( $\mathrm{H}$ and $\mathrm{PE}$ ) displayed significantly higher scores than clusters low in positive schizotypy (NS and L), which confirmed a link between positive schizotypy and increased creative skills. Interestingly, the fact that creative competencies appeared to be lower in nonschizotypal individuals (L group) than in $\mathrm{H}$ or PE was consistent with the hypothesis that positive schizotypy may have favorable facets, as proposed by others (Mohr \& Claridge, 2015).

When considering different dimensions of creative competencies (Epstein et al., 2008), results showed that Capturing (i.e., using dreams as sources of ideas, finding situations where new ideas can be observed), Broadening (i.e., seeking experience outside areas of expertise) and Surrounding (i.e., changing environments regularly, seeking out unusual stimuli) were preferentially associated with $\mathrm{H}$ and PE rather than with L and NS. This suggested that Capturing, Broadening and Surrounding were linked with high positive schizotypy (such as in $\mathrm{H}$ or PE), and this association appeared to be independent of negative schizotypy, considering that $\mathrm{H}$ and $\mathrm{PE}$ displayed different levels of negative schizotypy (high in $\mathrm{H}$ and low in PE). In contrast, Challenging (i.e., taking on difficult tasks, managing feelings associated with failure) was higher in PE than in L or NS, but also than in H. This indicated that increased Challenging was specific of the pure positive schizotypy cluster (PE). This suggested that positive schizotypy is linked with increased individuals' ability to take on difficult tasks or manage feeling linked with failure only when individuals also display low negative schizotypy. Indeed, when high positive schizotypy is associated with elevated negative schizotypy, such as in the $\mathrm{H}$ cluster, the level of Challenging is comparable to that of clusters low in positive schizotypy (i.e., L and NS). Of note, when considering each of these dimensions, all the scores of the pure positive schizotypy cluster (PE) were higher than L, which was consistent with the view that positive schizotypy has fortunate sides even when compared with non-schizotypal individuals (Mohr \& Claridge, 2015). 
In terms of creative cognitive processes (assessed with CPAC), this study showed that both $\mathrm{H}$ and PE scored significantly higher than NS, while PE scored higher than L. This suggested that individuals with elevated positive schizotypy (H or PE) display a higher level of cognitive processes associated with creativity, when compared with individuals characterized by negative schizotypy (NS). Moreover, the pure positive schizotypy cluster (PE) displayed a CPAC score significantly higher than the low schizotypy group (L), which confirmed an advantageous aspect of "healthy" schizotypy (defined as a high level of positive schizotypy with a low score of negative schizotypy).

When comparing clusters for the six subscales of creative cognitive processes, 4 out of 6 subscores (Idea manipulation, Imagery, Idea generation and Incubation) of the pure positive schizotypy cluster PE were significantly higher than NS, the negative schizotypy cluster. This supported the notion that implementation of several creative cognitive processes is more developed in positive than in negative schizotypy. Most interestingly, PE subscores regarding Imagery and Incubation were significantly more elevated than those of low schizotypes (L). This supported the view that positive schizotypy can be beneficial even when compared with nonschizotypal individuals. Of note, Imagery/sensory includes internal visualization and any type of sensory modality (auditory, tactile etc.) that can be connected to creativity, and it is thus a dimension specifically related to the hallucinatory aspect of schizotypy.

This study is based on self-report questionnaires, which could represent a limit, considering that individuals with high positive schizotypy might display a tendency to respond defensively, depending on the instructions they received to answer a survey (Mohr, Schofield, Leonards, Wilson, \& Grimshaw, 2018). However, this report also showed that presenting a survey as a creativity study, which is the case of our survey, minimizes defensive responses. In addition, inter-cluster differences in terms of creativity scale scores display small effect sizes, which represents another limit of our study. 


\section{References}

Abbott, G. R., Do, M., \& Byrne, L. K. (2012). Diminished subjective wellbeing in schizotypy is more than just negative affect. Personality and Individual Differences, 52(8), 914-918.

Batey, M., \& Furnham, A. (2008). The relationship between measures of creativity and schizotypy. Personality and Individual Differences, 45(8), 816-821.

Bronchain, J., Chabrol, H., \& Raynal, P. (2018). Could schizotypy protect against the negative outcomes of borderline traits on alcohol consumption? A cluster analytic study. Psychiatry research, 269, 21-24.

Cohen, A. S., \& Davis, T. E. (2009). Quality of life across the schizotypy spectrum: findings from a large nonclinical adult sample. Comprehensive Psychiatry, 50(5), 408-414.

Compton, M. T., Goulding, S. M., Bakeman, R., \& McClure-Tone, E. B. (2009). Confirmation of a four-factor structure of the Schizotypal Personality Questionnaire among undergraduate students. Schizophrenia Research, 111(1-3), 46-52.

Csikszentmihalyi, M. (2014). Society, Culture, and Person: A Systems View of Creativity. In M. Csikszentmihalyi (Ed.), The Systems Model of Creativity: The Collected Works of Mihaly Csikszentmihalyi (pp. 47-61). Dordrecht: Springer Netherlands. https://doi.org/10.1007/978-94-017-9085-7_4

Diener, E., Emmons, R. A., Larsen, R. J., \& Griffin, S. (1985). The Satisfaction with Life Scale. Journal of Personality Assessment, 49(1), 71-75.

Epstein, R., Schmidt, S. M., \& Warfel, R. (2008). Measuring and Training Creativity Competencies: Validation of a New Test. Creativity Research Journal, 20(1), 7-12. https://doi.org/10.1080/10400410701839876

Epstein, R., \& Phan, V. (2012). Which competencies are most important for creative expression?. Creativity Research Journal, 24(4), 278-282.

Ettinger, U., Mohr, C., Gooding, D. C., Cohen, A. S., Rapp, A., Haenschel, C., \& Park, S. (2015). Cognition and brain function in schizotypy: a selective review. Schizophrenia Bulletin, 
41(suppl_2), S417-S426.

Fisher, J. E., Heller, W., \& Miller, G. A. (2013). Neuropsychological differentiation of adaptive creativity and schizotypal cognition. Personality and Individual Differences, 54(1), 70-75.

Fonseca-Pedrero, E., Debbane, M., Ortuno-Sierra, J., Chan, R. C. K., Cicero, D. C., Zhang, L. C., ... Jablensky, A. (2017). The structure of schizotypal personality traits: a cross-national study. Psychol Med, 1-12. https://doi.org/10.1017/s0033291717001829

Goulding, A. (2004). Schizotypy models in relation to subjective health and paranormal beliefs and experiences. Personality and Individual Differences, 37(1), 157-167.

Keller, M. C., \& Miller, G. (2006). Resolving the paradox of common, harmful, heritable mental disorders: which evolutionary genetic models work best?. Behavioral and brain sciences, 29(4), 385-404.

Kwapil, T. R., \& Barrantes-Vidal, N. (2015). Schizotypy: Looking Back and Moving Forward. Schizophrenia Bulletin, 41, S366-S373.

McCreery, C., \& Claridge, G. (2002). Healthy schizotypy: the case of out-of-the-body experiences. Personality and Individual Differences, 32(1), 141-154.

Miller, A. L. (2014). A self-report measure of cognitive processes associated with creativity. Creativity Research Journal, 26(2), 203-218.

Miller, G. F., \& Tal, I. R. (2007). Schizotypy versus openness and intelligence as predictors of creativity. Schizophrenia Research, 93(1), 317-324.

Mohr, C., \& Claridge, G. (2015). Schizotypy-Do Not Worry, It Is Not All Worrisome. Schizophrenia Bulletin, 41(suppl 2), S436-S443.

Mohr, C., Schofield, K., Leonards, U., Wilson, M. S., \& Grimshaw, G. M. (2018). Psychiatric framing affects positive but not negative schizotypy scores in psychology and medical students. Psychiatry Research, 266, 85-89. https://doi.org/10.1016/j.psychres.2018.05.028

Mumford, M. D. (2003). Where Have We Been, Where Are We Going? Taking Stock in Creativity Research. Creativity Research Journal, 15(2-3), 107-120. 
https://doi.org/10.1080/10400419.2003.9651403

Nelson, B., \& Rawlings, D. (2010). Relating Schizotypy and Personality to the Phenomenology of Creativity. Schizophrenia Bulletin, 36(2), 388-399.

Nettle, D. (2006). Schizotypy and mental health amongst poets, visual artists, and mathematicians. Journal of Research in Personality, 40(6), 876-890.

Park, H. R. P., Kirk, I. J., \& Waldie, K. E. (2015). Neural correlates of creative thinking and schizotypy. Neuropsychologia, 73, 94-107.

Radel, R., Davranche, K., Fournier, M., \& Dietrich, A. (2015). The role of (dis)inhibition in creativity: Decreased inhibition improves idea generation. Cognition, 134, 110-120.

Raine, A., \& Benishay, D. (1995). The SPQ-B: A brief screening instrument for schizotypal personality disorder. Journal of Personality Disorders, 9(4), 346-355.

Raynal, P., Goutaudier, N., Nidetch, V., \& Chabrol, H. (2016). Typology of schizotypy in nonclinical young adults: Psychopathological and personality disorder traits correlates. Psychiatry Research, 246, 182-187.

Richards, R. (2001). Creativity and the schizophrenia spectrum: More and more interesting. Creativity Research Journal, 13(1), 111-132.

Ryff, C. D. (1989). Happiness Is Everything, or Is It - Explorations on the Meaning of Psychological Well-Being. Journal of Personality and Social Psychology, 57(6), 10691081.

Sass, L. A., \& Schuldberg, D. (2001). Introduction to the special issue: Creativity and the schizophrenia spectrum. Creativity Research Journal, 13(1), 1-4.

Schofield, K., \& Claridge, G. (2007). Paranormal experiences and mental health: Schizotypy as an underlying factor. Personality and Individual Differences, 43(7), 1908-1916.

Tabak, N. T., \& de Mamani, A. G. W. (2013). Latent profile analysis of healthy schizotypy within the extended psychosis phenotype. Psychiatry Research, 210(3), 1008-1013.

Tsakanikos, E., \& Claridge, G. (2005). More words, less words: verbal fluency as a function of 
'positive'and 'negative'schizotypy. Personality and Individual Differences, 39(4), 705713.

van Os, J., \& Linscott, R. J. (2012). Introduction: The Extended Psychosis PhenotypeRelationship With Schizophrenia and With Ultrahigh Risk Status for Psychosis. Schizophrenia Bulletin, 38(2), 227-230.

Wang, L., Long, H., Plucker, J. A., Wang, Q., Xu, X., \& Pang, W. (2018). High Schizotypal Individuals Are More Creative? The Mediation Roles of Overinclusive Thinking and Cognitive Inhibition. Frontiers in Psychology, 9. https://doi.org/10.3389/fpsyg.2018.01766 Zigmond, A. S., \& Snaith, R. P. (1983). The Hospital Anxiety and Depression Scale. Acta Psychiatrica Scandinavica, 67(6), 361-370. 


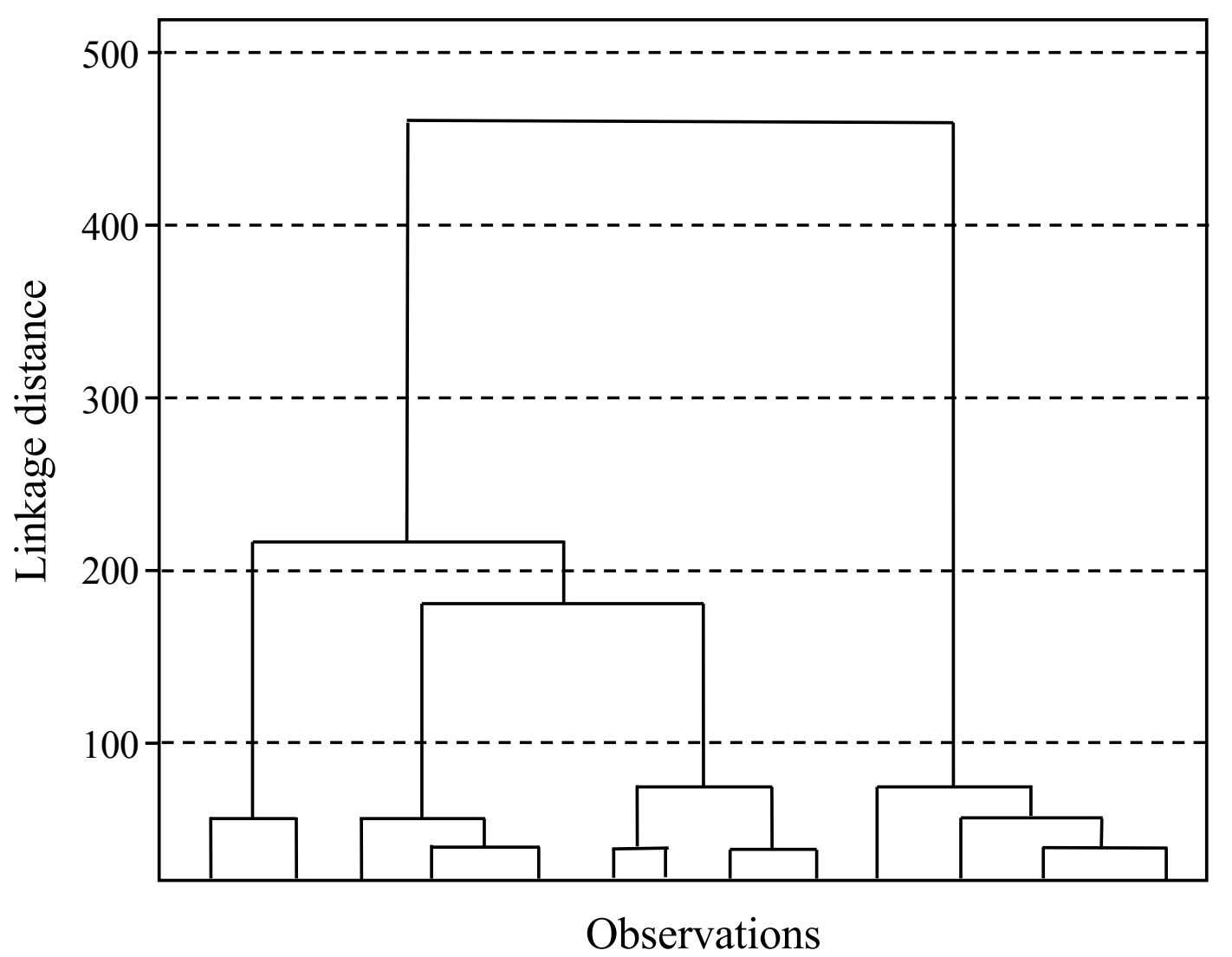

Figure 1. Hierarchical clustering dendrogram 


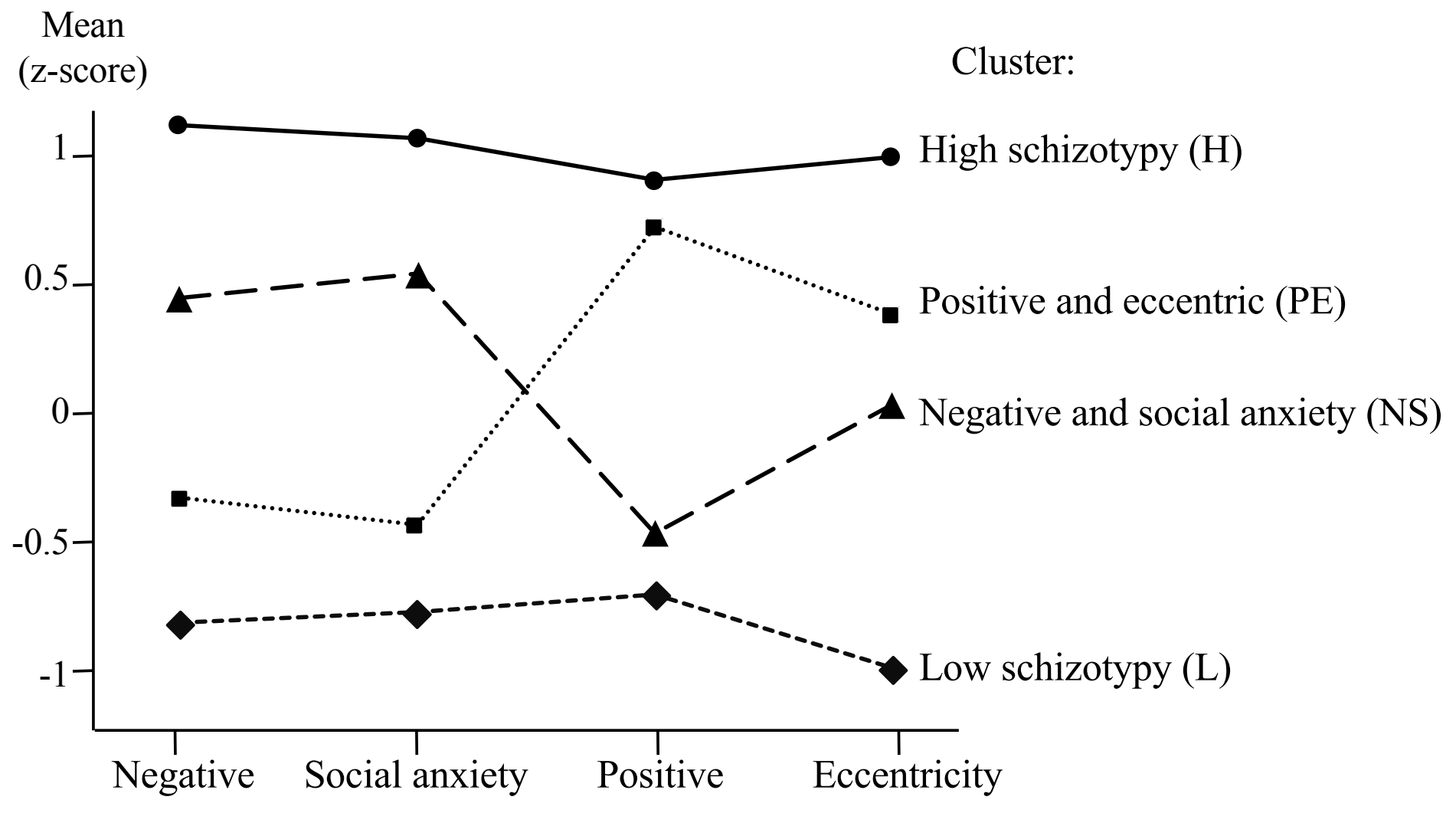

Figure 2. Four-cluster solution based on the four factors of schizotypal traits indicated on the $\mathrm{x}$-axis. 
Table 1

Descriptive statistics and typology of individuals on 4 dimensions of schizotypy: differences in creativity scales and psychopathology

(cluster comparison using ANOVA and Tukey post hoc test)

\begin{tabular}{|c|c|c|c|c|c|c|c|c|c|c|c|}
\hline & Sample & Range & $95 \%$ & Cron- & Cluster & $M(S D)$ & & & & & \\
\hline & $\begin{array}{l}N=946 \\
M(S D)\end{array}$ & & $\begin{array}{c}\text { confidence } \\
\text { interval }\end{array}$ & $\begin{array}{l}\text { bach's } \\
\text { alpha s }\end{array}$ & $\begin{array}{c}\text { High } \\
\text { schizotypy }(\mathrm{H}) \\
n=174 \quad 18 \%\end{array}$ & $\begin{array}{c}\text { Low } \\
\text { schizotypy (L) } \\
n=237 \quad 25 \%\end{array}$ & $\begin{array}{c}\text { Positive \& } \\
\text { eccentric (PE) } \\
n=25527 \%\end{array}$ & $\begin{array}{c}\text { Negative \& social } \\
\text { anxiety (NS) } \\
n=280 \quad 30 \%\end{array}$ & $F$ & $\begin{array}{l}\text { Significant } \\
\text { comparisons }\end{array}$ & $\begin{array}{c}\text { Eta } \\
\text { squa- } \\
\text { red }\end{array}$ \\
\hline Schizotypy SPQ-B & $53.08(11.7)$ & $22-84$ & $52.33-53.83$ & .88 & $69.36(5.86)$ & $38.69(6.33)$ & $52.99(5.98)$ & $55.22(5.51)$ & $918.56^{*}$ & $\mathrm{~L}<\mathrm{PE}<\mathrm{NS}<\mathrm{H}$ & .74 \\
\hline Positive schizotypy & $8.49(2.92)$ & $4-16$ & $8.31-8.68$ & & $11.12(2.38)$ & $6.2(1.83)$ & $10.56(2.14)$ & $6.93(1.79)$ & $346.6^{*}$ & $\mathrm{~L}<\mathrm{NS}<\mathrm{PE}<\mathrm{H}$ & .52 \\
\hline Negative schizotypy & $12.65(3.45)$ & $5-20$ & $12.43-12.87$ & & $16.52(2.32)$ & $9.53(2.41)$ & $11.29(2.43)$ & $14.11(2.31)$ & $354.67 *$ & $\mathrm{~L}<\mathrm{PE}<\mathrm{NS}<\mathrm{H}$ & .53 \\
\hline Social anxiety & $9.9(3.01)$ & $4-16$ & $9.71-10.09$ & & $13.13(1.99)$ & $7.32(2.19)$ & $8.38(1.99)$ & $11.46(1.98)$ & $374.78^{*}$ & $\mathrm{~L}<\mathrm{PE}<\mathrm{NS}<\mathrm{H}$ & .54 \\
\hline Eccentricity & $7.49(2.47)$ & $3-12$ & $7.34-7.65$ & & $9.94(1.62)$ & $4.81(1.58)$ & $8.36(1.8)$ & $7.46(1.87)$ & $324.81^{*}$ & $\mathrm{~L}<\mathrm{NS}<\mathrm{PE}<\mathrm{H}$ & .51 \\
\hline Creativ. compet. ECCIi & $90.71(13.62)$ & $49-132$ & $89.84-91.58$ & .81 & $93.05(14.58)$ & $87.69(13.09)$ & $95.96(11.91)$ & $87.03(13.14)$ & $27.11 *$ & $\mathrm{~L}, \mathrm{NS}<\mathrm{H}, \mathrm{PE}$ & .08 \\
\hline Creativ. cognit. CPAC & $104.41(14.76)$ & $32-130$ & $\begin{array}{l}\text { IUS.4/- } \\
10525\end{array}$ & .9 & $106.32(16.1)$ & $103.07(14)$ & $\begin{array}{l}10 / .10 \\
615811\end{array}$ & $101.85(13.22)$ & $7.66^{*}$ & $\mathrm{NS}<\mathrm{H}, \mathrm{PE} \mathrm{L}<\mathrm{PE}$ & .03 \\
\hline Subjective well-being & $90.23(14.39)$ & $34-124$ & $89.31-91.14$ & .88 & $77.68(14.19)$ & $99.03(10.35)$ & $96.55(11.62)$ & $84.82(11.54)$ & $153.09^{*}$ & $\mathrm{H}<\mathrm{NS}<\mathrm{L}, \mathrm{PE}$ & .33 \\
\hline Life satisfaction & $23.95(6.26)$ & $5-35$ & $23.55-24.34$ & .82 & $19.96(6.89)$ & $26.12(5.78)$ & $25.64(5.14)$ & $23.04(5.8)$ & $47.34 *$ & $\mathrm{H}<\mathrm{NS}<\mathrm{L}, \mathrm{PE}$ & .13 \\
\hline Anxiety & $9.14(3.9)$ & $1-21$ & 8.9-9.8 & .78 & $12.22(3.7)$ & $7.32(3.18)$ & $8.51(3.4)$ & $9.35(3.84)$ & $67.92 *$ & $\mathrm{~L}<\mathrm{PE}<\mathrm{NS}<\mathrm{H}$ & .18 \\
\hline Depressive symptoms & $4.69(2.96)$ & $0-19$ & $4.5-4.88$ & .65 & $6.53(3.3)$ & $3.37(2.5)$ & $3.84(2.32)$ & $5.43(2.8)$ & $60.94 *$ & $\mathrm{~L}, \mathrm{PE}<\mathrm{NS}<\mathrm{H}$ & .16 \\
\hline
\end{tabular}

$* p<.05$ 
Table 2

Principal component analysis of 22 SPQ-B items

\begin{tabular}{|c|c|c|c|c|}
\hline \multirow[b]{2}{*}{ item } & \multicolumn{4}{|c|}{ Factor loadings } \\
\hline & $\begin{array}{c}1 \\
\text { Negative } \\
\text { schizotypy }\end{array}$ & $\begin{array}{c}2 \\
\text { Social } \\
\text { anxiety }\end{array}$ & $\begin{array}{c}3 \\
\text { Positive } \\
\text { schizotypy }\end{array}$ & $\begin{array}{c}4 \\
\text { Eccentricity }\end{array}$ \\
\hline 1. people find me aloof and distant & .48 & .26 & -.15 & .42 \\
\hline 2. sense some person or force & .12 & -.13 & $\underline{.68}$ & .21 \\
\hline 3. unusual mannerisms and habits & .15 & .16 & .24 & .71 \\
\hline 4. people can tell what you're thinking & .18 & .32 & .54 & -.02 \\
\hline 5. noticed special signs for you & .15 & .04 & .7 & .1 \\
\hline 6. people think I am very bizarre & .15 & .23 & .22 & .78 \\
\hline 7. on my guard even with friends & .74 & .15 & .2 & .04 \\
\hline 8. people find me vague and elusive & .19 & $\underline{.5}$ & .27 & .28 \\
\hline 9. often pick up hidden threats & .48 & .19 & .27 & .22 \\
\hline 10. people are taking notice of you & .31 & .45 & .29 & .02 \\
\hline 11. discomfort with unfamiliar people & .26 & .78 & .03 & .06 \\
\hline 12. astrology, UFOs, ESP, sixth sense & .05 & -.12 & .61 & .2 \\
\hline 13. I use words in unusual ways & -.07 & .38 & .36 & .12 \\
\hline 14. not let people know about you & .71 & .15 & .07 & .17 \\
\hline 15. tend to keep in the background & .37 & $\underline{.66}$ & -.11 & .2 \\
\hline 16. distracted by distant sounds & .23 & .14 & .49 & .34 \\
\hline 17. stops people from taking advantage & .53 & .12 & .38 & .01 \\
\hline 18. unable to get "close" to people & $\underline{.6}$ & .3 & .01 & .22 \\
\hline 19. I am an odd, unusual person & .11 & .01 & .22 & .75 \\
\hline 20. hard to communicate clearly & .41 & .49 & .11 & .31 \\
\hline 21. very uneasy talking to people & .27 & .78 & -.06 & .06 \\
\hline 22. tend to keep my feelings to myself & $\underline{.62}$ & .18 & -.09 & .06 \\
\hline Eigenvalues & $\overline{6.56}$ & 2.33 & 1.28 & 1.21 \\
\hline Explained variance $(\%)$ & 15.06 & 13.59 & 11.91 & 11.14 \\
\hline
\end{tabular}

Values $>.3$ are in bold

Items retained in the model are underlined 
Table 3

Cluster comparison regarding distinct dimensions of creativity competencies and creative cognitive processes (using ANOVA and Tukey post hoc test)

\begin{tabular}{|c|c|c|c|c|c|c|c|c|c|}
\hline & Sample & $95 \%$ & Cluster $M(S D)$ & & & & & & \\
\hline & $N=946$ & $\begin{array}{l}\text { confidence } \\
\text { interval }\end{array}$ & $\begin{array}{c}\text { High } \\
\text { schizotypy (H) }\end{array}$ & $\begin{array}{c}\text { Low } \\
\text { schizotypy (L) }\end{array}$ & $\begin{array}{c}\text { Positive \& } \\
\text { eccentric (PE) }\end{array}$ & $\begin{array}{l}\text { Negative \& social } \\
\text { anxiety (NS) }\end{array}$ & $F$ & $\begin{array}{l}\text { Significant } \\
\text { comparisons }\end{array}$ & $\begin{array}{l}\text { Eta } \\
\text { squa- }\end{array}$ \\
\hline & $M(S D)$ & & $n=174 \quad 18 \%$ & $n=23725 \%$ & $n=25527 \%$ & $n=280 \quad 30 \%$ & & & red \\
\hline \multicolumn{10}{|l|}{ Creative competencies ECCIi } \\
\hline Capturing & $21.05(5.47)$ & $20.7-21.4$ & $22.99(5.4)$ & $19.21(5.27)$ & $22.52(5.12)$ & $20.06(5.26)$ & $27.5^{*}$ & $\mathrm{~L}, \mathrm{NS}<\mathrm{H}, \mathrm{PE}$ & .09 \\
\hline Challenging & $22.12(4.14)$ & $21.85-22.38$ & $21.29(4.55)$ & $22.14(4.06)$ & $23.29(4.02)$ & $21.54(3.81)$ & $11.31^{*}$ & $\mathrm{~L}, \mathrm{H}, \mathrm{NS}<\mathrm{PE}$ & .04 \\
\hline Broadening & $28.34(4.41)$ & $28.06-28.62$ & $29.06(4.33)$ & $27.86(4.25)$ & $29.3(3.83)$ & $27.43(4.84)$ & $10.84 *$ & $\mathrm{~L}, \mathrm{NS}<\mathrm{H}, \mathrm{PE}$ & .03 \\
\hline Surrounding & $19.21(5.83)$ & $18.83-19.58$ & $19.71(6.05)$ & $18.49(6)$ & $20.85(5.41)$ & $18(5.54)$ & $12.94 *$ & $\mathrm{~L}, \mathrm{NS}<\mathrm{PE} \quad \mathrm{NS}<\mathrm{H}$ & .04 \\
\hline \multicolumn{10}{|l|}{ Creative cognit. proc. CPAC } \\
\hline Idea manipulation & $19.93(3.5)$ & $19.7-20.15$ & $20.34(3.72)$ & $19.67(3.38)$ & $20.45(3.63)$ & $19.41(3.25)$ & $5.25^{*}$ & $\mathrm{NS}<\mathrm{H}, \mathrm{PE}$ & .02 \\
\hline Imagery & $22.14(4.21)$ & $21.87-22.41$ & $22.37(4.49)$ & $21.77(4.13)$ & $22.98(4.33)$ & $21.55(3.88)$ & $6^{*}$ & $\mathrm{~L}, \mathrm{NS}<\mathrm{PE}$ & .02 \\
\hline Flow & $15.64(3.25)$ & $15.44-15.85$ & $16.13(3.26)$ & $15.5(3.33)$ & $15.76(3.1)$ & $15.36(3.18)$ & 2.24 & & \\
\hline Metaphoric/analogic think. & $15.08(2.91)$ & $14.9-15.27$ & $15.37(3.1)$ & $14.97(3.04)$ & $15.25(2.94)$ & $14.85(2.63)$ & 1.56 & & \\
\hline Idea generation & $22.05(3.52)$ & $21.82-22.27$ & $21.95(3.82)$ & $22.09(3.45)$ & $22.64(3.59)$ & $21.52(3.23)$ & $4.63^{*}$ & $\mathrm{NS}<\mathrm{PE}$ & .015 \\
\hline Incubation & $9.57(2.45)$ & $9.41-9.72$ & $10.16(2.8)$ & $9.06(2.32)$ & $10.08(2.32)$ & $9.16(2.27)$ & $13.51 *$ & $\mathrm{~L}, \mathrm{NS}<\mathrm{H}, \mathrm{PE}$ & .041 \\
\hline
\end{tabular}

$* p<.05$ 